\title{
Search Strategy Optimization for Intruder Detection
}

\author{
Rensheng Wang and Jeffrey V. Nickerson
}

\begin{abstract}
Sensors can take advantage of environmental information to sweep for a moving intruder in a manner that will look random to an intruder, but that will maximize the probability of detection. We show how likelihood information about intrusion from particular sectors can be used to design a sweep strategy.
\end{abstract}

Index Terms-Intrusion detection, search theory.

\section{INTRODUCTION}

$\mathbf{I}$ NTRUDERS are constrained by the environment, and such constraints can be used to tailor a sweeping strategy for a fixed-position set of sensors. In many environments, sensors (for example, cameras) sweep the surroundings in a back and forth manner, but if an attack is more likely on the edges than through the center, such a search strategy is wasteful.

We assume that sensors are listening for an intruder's activity, and need to focus on a sector of space many seconds in order to detect an intrusion. A full sweep of an area may not be possible - by the time the field is swept, an intruder may have already reached the target and left. In many cases, however, environmental conditions give us information about the likelihood of attack: wind direction or river currents may make certain trajectories of attack improbable. We build on the ideas discussed in search theory [1], [2].

\section{PROPOSED SCHEME}

Consider a general scenario where we have multiple sensors monitoring $M$ partitioned areas, and each sensor is assigned with a selection of multiple sectors from $M$. If every two sensors have no overlapping sweeping areas, this multisensor multisector arrangement reduces to a set of independent single-sensor multisector situations. We next focus our discussion on these single sensor situations, and then briefly discuss how the solutions can be extended to situations where sensors overlap their sweep fields. Assume that we have in total $N$ sectors assigned for one sensor. We need to determine the frequency of sensor detection for each sector, $f(i)$, with given knowledge of the probability $P(i)$ for the intruder appearing in each sector (see Fig. 1). Here, $f(i)$ indicates how often this sensor will visit the sector " $i$." The problem of interest is how to make use of the optimal search strategy to adjust the frequency selections $f(i)$. We base our analysis on the worst-case performance for intruder detection.

Manuscript received July 7, 2006; revised September 19, 2006; accepted September 26, 2006. This work was supported by the Office of Naval Research under Grant N00014-05-1-00632. The associate editor coordinating the review of this paper and approving it for publication was Prof. Denise Wilson.

The authors are with the Stevens Institute of Technology, Hoboken, NJ 07030

USA (e-mail: rwang1@ stevens.edu; jnickerson@ stevens.edu).

Digital Object Identifier 10.1109/JSEN.2006.890161

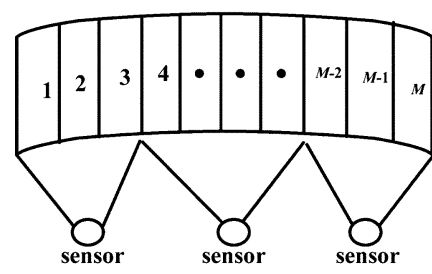

(a)

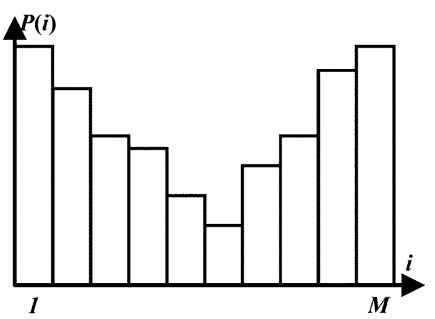

(b)
Fig. 1. (a) Sector assignments for sensors. (b) Associated intruder probability.

\section{A. Maximum Intrusion Distance}

Without loss of generality, let us assume sector " $i_{0}$ " has the lowest frequency for sensor detection. The maximum intrusion time for the sector " $i_{0}$ " is the time gap between two adjacent sensor detection happenings, $t / f\left(i_{0}\right)$, where $t$ is the time duration a sensor spends on one sector. Denoting the intruder speed $S p$ a constant, we have the maximum distance $S p \times t / f\left(i_{0}\right)$ an intruder can take during this period.

\section{B. Minimum Maximum Intrusion Distance}

To minimize the maximum intrusion distance $S p \times t / f\left(i_{0}\right)$, we need to keep increasing $f\left(i_{0}\right)$ until $f\left(i_{0}\right)$ is no longer the smallest frequency. This minimization ends with an extreme situation where $f\left(i_{0}\right)=1 / N$

$$
\min _{f\left(i_{0}\right)} S p \times t / f\left(i_{0}\right)=S p \times t /(1 / N)=S p \times N \times t .
$$

Equation (1) implies that all the sectors are equally treated with identical detection frequencies $f(i)=1 / N$.

\section{Minimum Average Maximum Intrusion Distance}

Bearing in mind the intruder has different probabilities in different sectors, the average maximum intrusion distance is

$$
\sum_{i=1}^{N} P(i) \times S p \times t / f(i)=S p \times t \sum_{i=1}^{N} \frac{P(i)}{f(i)} .
$$

The optimal detection frequencies $\{f(i)\}$ is obtained by minimizing the average maximum intrusion distance

$$
\{f(i)\}=\arg \min _{f(i)} S p \times t \sum_{i=1}^{N} \frac{P(i)}{f(i)} \text {, s.t. } \sum_{i=1}^{N} f(i)=1 .
$$

Equation (3) can be solved using a Lagrange multiplier with cost function $V(f(i), \lambda)=\sum_{i=1}^{N} P(i) / f(i)+\lambda\left(\sum_{i=1}^{N} f(i)-1\right)$, where $\lambda$ is the Lagrange multiplier. Differentiation of $V(f(i), \lambda)$ with respect to $\{f(i)\}$ gives the optimal solution. $\partial V(f(i), \lambda) / \partial f(i)=-P(i) / f^{2}(i)+\lambda=0$, for $i=1, \cdots, N$. The above set of equations yield

$$
\{f(i)\}=\alpha\{\sqrt{P(i)}\}
$$




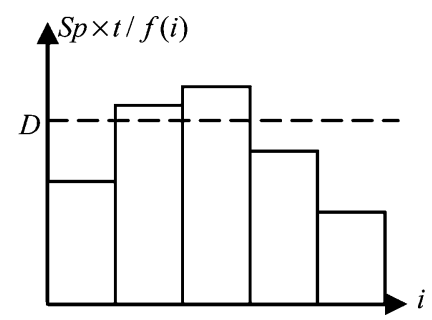

(a)

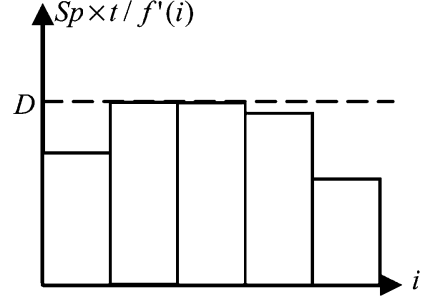

(b)
Fig. 2. Optimization of searching frequency $f(i)$ with respect to tolerance range $D$ when $S p \times t \times N<D<S p \times t / f\left(i_{0}\right)$ : (a) before; (b) after.

where $\alpha$ is a scaling factor and can be determined as $\alpha=$ $1 / \sum_{i=1}^{N} \sqrt{P(i)}$ [refer to the constraint in (3)].

Although an optimal searching solution is given in (4), in reality, a natural concern is how far the intruder can approach before we perceive its existence. We introduce the tolerance range $D$, the maximum distance we can tolerate before the intruder is detected by the sensor. We next investigate the optimal searching strategy with respect to this new constraint.

1) $D \geq S p \times t / f\left(i_{0}\right)$.

The largest intrusion distance is within the tolerance range. The solution in (4) is optimal in the sense that the average maximum detection distance is minimized.

2) $D<S p \times t / f\left(i_{0}\right)$.

The largest intrusion distance is out of the tolerance range, and, therefore, we will have some possibility of failing to detect. In order to avoid this and satisfy the tolerance range $D$, we need to redetermine the sensor searching frequency sequence $\left\{f^{\prime}(i)\right\}$. Denote " $\left\{i_{m}\right\}$ " the index sequence for sectors where the maximum intrusion distance is larger than the tolerance range $D$, and " $\left\{i_{c}\right\}$ " the complement index set. To satisfy the tolerance range, we need make the frequency set related with " $\left\{i_{m}\right\}$ " be $f^{\prime}(j)=S p \times t / D, j \in\left\{i_{m}\right\}$, i.e., the intrusion distance equals to $D$, and redetermine the residual complement frequency set as follows:

$$
\begin{aligned}
\left\{f^{\prime}(l)\right\} & =\arg \min _{f^{\prime}(l)} S p \times t \sum_{l \in\left\{i_{c}\right\}} \frac{P(l)}{f^{\prime}(l)} \\
\text { s.t. } \sum_{l \in\left\{i_{c}\right\}} f^{\prime}(l) & =1-\sum_{j \in\left\{i_{m}\right\}} f^{\prime}(j)
\end{aligned}
$$

where we are minimizing the average maximum intrusion distance over the residual complement sectors [see Fig. 2(b)]. It is easy to see that the solution is similar to that of (3). From Fig. 2, we can see that the redetermination of sensor detection frequencies essentially reshapes the intrusion distance curve within the tolerance range, i.e., makes the curve below the boundary $D$. Therefore, the area that the curve $S p \times t / f^{\prime}(i)$ covers should be less than what the tolerance boundary covers

$$
\sum_{i=1}^{N} S p \times t / f^{\prime}(i) \leq \sum_{i=1}^{N} D=N D .
$$

For a given constraint $\sum_{i=1}^{N} f^{\prime}(i)=1$, using the Schwartz inequality, we have $\sum_{i=1}^{N} 1 / f^{\prime}(i)=$

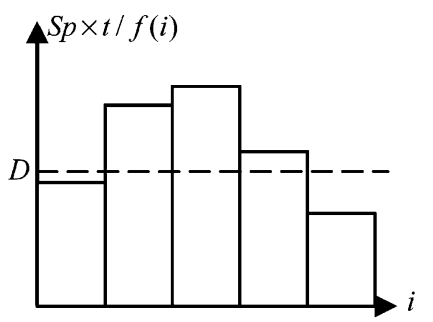

(a)

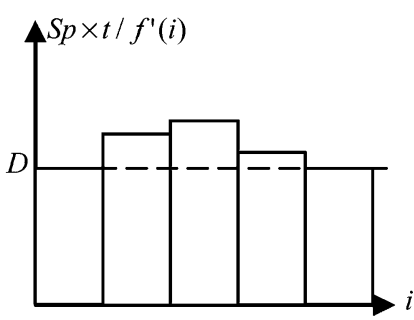

(b)
Fig. 3. Optimization of searching frequency $f(i)$ with respect to tolerance range $D$ when $S p \times t / f\left(i_{N}\right)<D<S p \times t \times N$ : (a) before; (b) after.

$\left(\sum_{i=1}^{N} f^{\prime}(i)\right)\left(\sum_{i=1}^{N} 1 / f^{\prime}(i)\right) \geq N^{2}$ Inserting the above inequality to (6), we have $S p \times t \times N^{2} \leq N D$. Then, the tolerance range $D$ has to satisfy

$$
D \geq S p \times t \times N
$$

Remarks:

R1) When $D=S p \times t \times N$, it reduces to $B$ ) minimum maximum intrusion distance case.

R2) Consider when $S p \times t / f\left(i_{N}\right)<D<S p \times t \times N$, where $i_{N}$ is the sector index with largest frequency (i.e., smallest intrusion distance). We denote " $\left\{i_{m}\right\}$ " the index sequence for sectors where the maximum intrusion distance is less than $D$, and " $\left\{i_{c}\right\}$ " the complement index set. We set $f^{\prime}(j)=S p \times t / D, j \in$ $\left\{i_{m}\right\}$. Then $\left\{f^{\prime}(l)\right\}, l \in\left\{i_{c}\right\}$ is determined by (5) [see Fig. 3].

R3) When $D<S p \times t / f\left(i_{N}\right)$, there is nothing we can do about the minimum average maximum intrusion distance since the tolerance range is too short for any tuning work.

Now consider the case where sensor sweep fields overlap. For example, if sector " $i$ " is appointed with three different sensors, we modify the intrusion probability with sector " $i$ " to $P(i) / 3$ for each sensor. (In future work, we will analyze the simultaneous events $\prod_{k} f_{k}(i)$, where $f_{k}(i)$ denotes the searching frequency for sector $i$ with the $k$ th sensor). In other words, we can approximate overlapping sectors as nonoverlapping sectors for every single sensor with divided probability $P(i)$, thereby reducing the problem of overlapping fields to one of independent fields.

\section{CONCLUSION}

We developed an optimal search strategy with respect to the minimum average maximum distance for intruder detection, when the intruder intrusion probabilities over certain areas are unevenly distributed. This work has applications to a wide set of problems in which environmental constraints are known.

\section{REFERENCES}

[1] L. D. Stone, Theory of Optimal Search, 2nd ed. Arlington, VA: Oper. Res. Soc. Amer., 1989.

[2] S. Alpern and S. Gal, The Theory of Search Games and Rendezvous. New York: Kluwer, 2002. 